\title{
Fast Solid State Electron Detectors Based on the Principle of Silicon Drift Detectors for Efficient Soft and Hard Matter Analysis
}

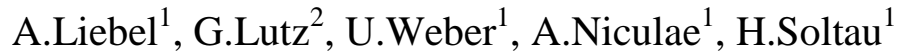 \\ ${ }^{1}$ PNDetector GmbH, Otto-Hahn-Ring 6, D-81739 München, Germany \\ ${ }^{2}$ PNSensor GmbH, Otto-Hahn-Ring 6, D-81739 München, Germany
}

Fast imaging with electrons is a field of growing interest. Major reasons for high scan rates are the investigation of sensitive soft matter specimen (to minimize sample damage) or the growing demand on high throughput in industrial applications. Modern microscopes will need advanced detectors which are fast even under difficult imaging conditions. For Backscattered Electron (BSE) imaging or Scanning Transmission Electron Microscopy (STEM) most instruments use solid state (silicon) or scintillator detectors. The two major benefits of solid state detectors are the slim shape and the freedom in designing any preferred geometry. However, they have to be large to capture enough signal, but since the size of the detector also determines the signal capacitance the speed of the detector system at high signal amplification is limited. Hence, new readout concepts have to be implemented in order to accomplish solid state electron detectors which combine large active areas and high detection speed.

Last year we introduced a new detector concept based on the principle of Silicon Drift Detectors (SDD) with integrated Field Effect Transistor (FET) [1]. These sensors are well known from Energy Dispersive X-ray (EDX) spectroscopy. They make use of the principle of sideward depletion to collect the signal charge from a large detector volume to a small anode with very low signal capacitance. Instead of detecting single X-ray photons, SDDs can in principle also be used to measure dynamic electron currents. However, in order to manage the wide variety of electron current intensities an additional feature has to be added in order to control the current amplification. In our new detector concept this is done by a second integrated FET which is connected to the anode. It can be used to regulate the mechanism of discharging the anode and therefore to adjust the gain to the signal intensity. That way, a new electron current detector has been created that features an extremely small signal capacitance (and therefore low detector noise) and a very fast first amplification stage integrated into the chip.

Test samples of the new detector type have been fabricated with active areas up to $8 \mathrm{~mm}^{2}$ and measurements with these structures were performed inside the SEM. Figure 1 shows an $8 \mathrm{~mm}^{2}$ test detector installed inside the microscope in a configuration perpendicular to the beam axis. Figure 2a presents the measurement result of this structure where the maximum detector speed was determined by fast scanning over a knife edge. The achieved minimum signal rise time at $50 \mathrm{kOhm}$ gain was well below 50 nsec which enables scanning with pixel dwell times of 50 nsec or less without the occurance of smearing effects. Increasing the gain reduces the detection speed (due to the small parasitic capacitance of the first amplification stage the signal rise time) but is still below 500 nsec for very high gain values of $10 \mathrm{MOhm}$ (see Figure 2b). This is about 10 times faster than conventional BSE detectors under similar conditions. This allows for high scan rates even at very weak electron signals which is very beneficial, especially for soft matter analysis. Fast measurements with low primary currents and energies can reduce measurement time, sample damage and therefore preparation time. 
Finally, we fabricated the first larger annular BSE detector chip based on SDD technology. It has a total active area of $40 \mathrm{~mm}^{2}$ and will be soon integrated and tested in an SEM. We will present measurements results and BSE images with the new detector that demonstrate its potential for fast and non-destructive imaging of soft and hard matter samples.

[1] A.Liebel et al., Microscopy \& Microanalysis, vol. 20, S3 (2014), pp. 28-29

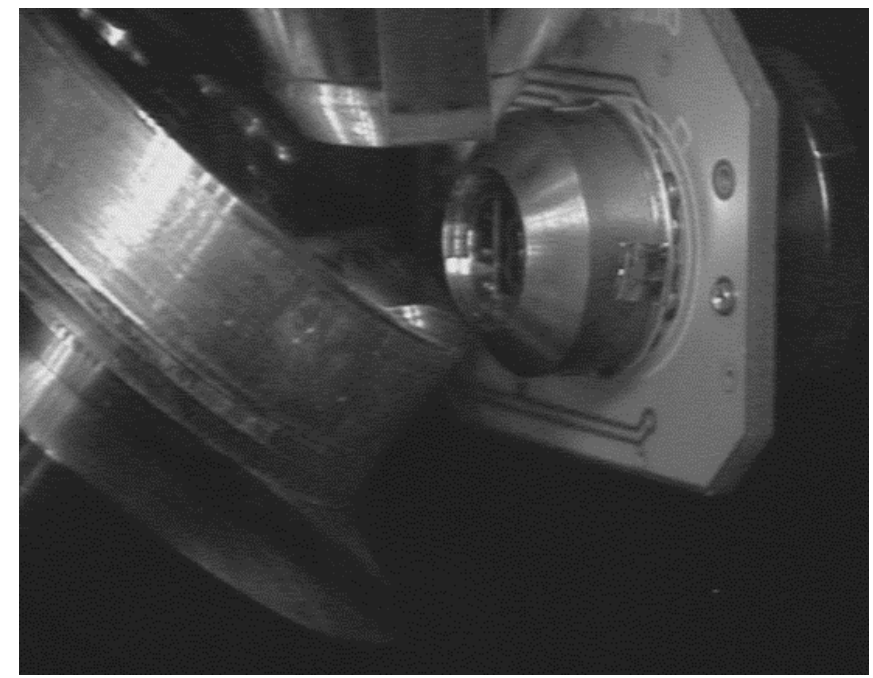

Figure 1. Test detector fabricated last year with $8 \mathrm{~mm}^{2}$ active area inside the SEM. Measurement results of a larger new detector in annular configuration will be shown.

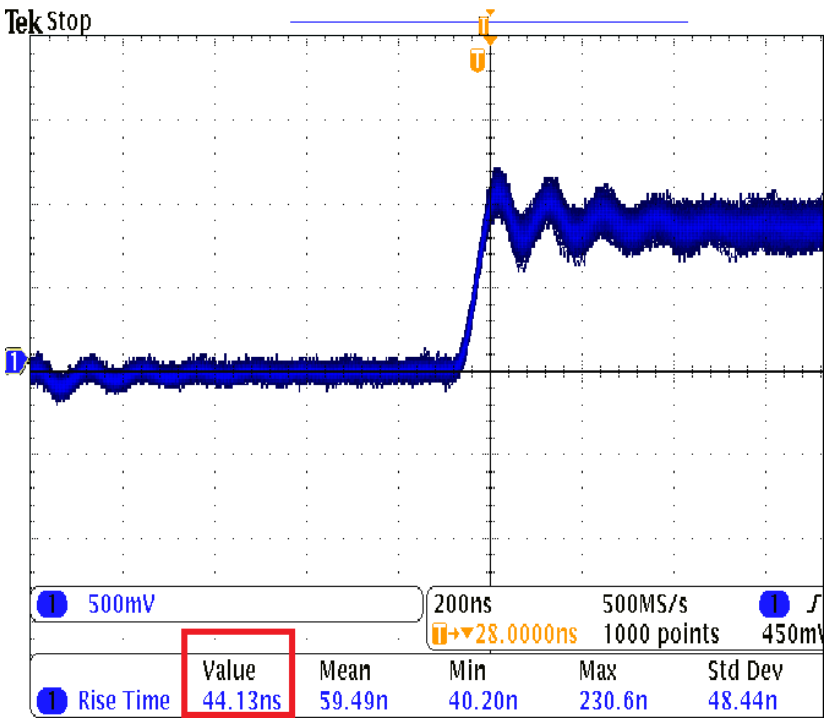

a)

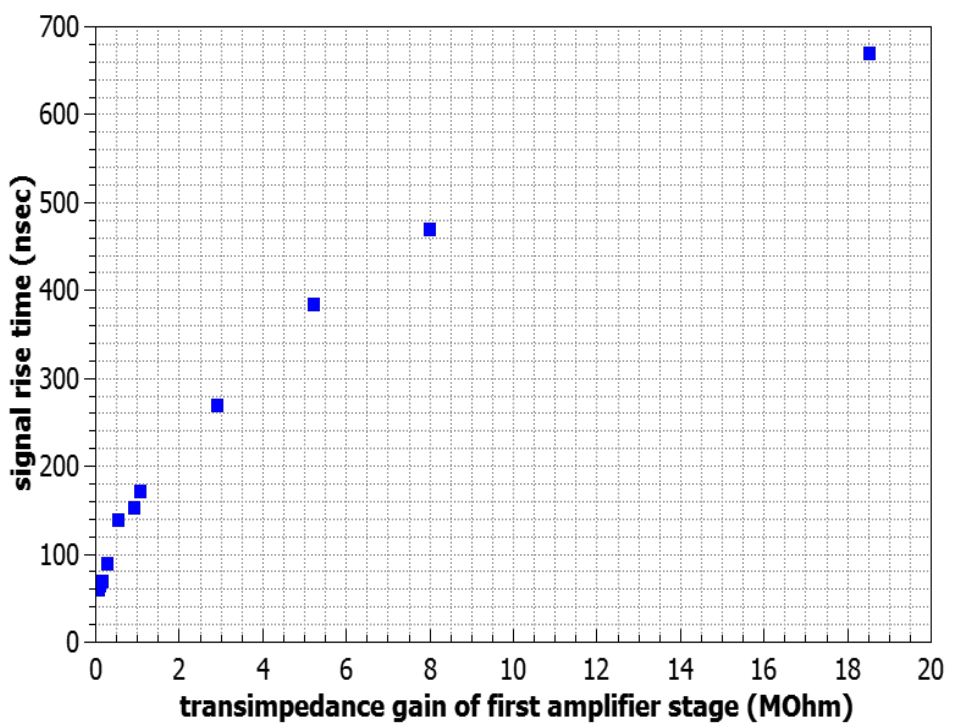

b)

Figure 2. a) By fast scanning over a knive edge signal rise times of less than 50 nsec could be achieved with an $8 \mathrm{~mm}^{2}$ test detector at $50 \mathrm{KOhm}$ first stage gain. b) The measured rise time is still $<500 \mathrm{nsec}$ at a very high gain of $10 \mathrm{MOhm}$ as needed for weak signals which are typical for soft matter analysis. This is about 10 times faster than a conventional solid state BSE detector at similar gain. 